\title{
Enabling Live Dialogic and Collaborative Learning between Field and Indoor Contexts
}

\author{
Tim Coughlan, Anne Adams \\ Institute of Educational Technology \\ The Open University \\ Milton Keynes, MK7 6AA \\ \{tim.coughlan,a.adams\}@open.ac.uk
}

\author{
Yvonne Rogers \\ Pervasive Interaction Lab \\ The Open University \\ Milton Keynes, MK7 6AA \\ y.rogers@open.ac.uk
}

\author{
Sarah-Jane Davies \\ Dept. of Earth \& Environmental Sciences \\ The Open University \\ Milton Keynes, MK7 6AA \\ s.j.m.davies@open.ac.uk
}

\begin{abstract}
This paper explores how field and indoor based students can be connected so that their contrasting circumstances and capabilities are used as a basis for learning. We describe the design of the 'Out There and In Here' system and activity. Using naturalistic evaluations in the context of higher education earth science, we find evidence that this approach can be beneficial in developing essential skills, by supporting dialogue and collaboration across diverse contexts. This provokes novel forms of reflection and motivation, and could inspire a new generation of learning tools combining mobile and collaborative technologies. We discuss important issues in this design space, such as asymmetrical dependencies and structures for dialogic and collaborative learning.
\end{abstract}

\section{Education, Mobile. Tabletop. Science. Fieldwork. Communication. Collaboration. Dialogic.}

\section{INTRODUCTION}

As communications technologies extend their reach and capabilities, possibilities emerge for new forms of human-human interactions linking diverse locations. In this research we consider that the contrast in the circumstances and capabilities of distanced users could be exploited in novel and valuable ways for education. For example, mobile learning research has shown that students in fieldwork inquiries can benefit from having digital information resources to hand, or by providing support to perform analysis tasks in situ. However, cost and time constraints on fieldwork, coupled with a desire to engage students with the field environment, rather than a computer screen, could be used as arguments that this approach is inefficient. Meanwhile, students working indoors could benefit from live interaction with a fieldwork context to ground their understanding. Ubiquitous computing technologies present opportunities for supporting these forms of dialogue and collaboration which are yet to be well utilised.

New technologies have not only altered how we learn, but also what it is important to learn. With vast amounts of information at our fingertips, communication, collaboration, and inquiry skills, alongside the ability to effectively utilise new technologies, are becoming prioritised above the rote learning of facts. Enabling these learning outcomes requires the development and adoption of radically different approaches to education, that themselves utilise new technologies (e.g. Wegerif, 2007, Mcwhaw et al 2003, Rochelle \& Pea, 2002). In this paper we explore how these kinds of novel juxtapositions of learning experiences can be enabled, and the potential they hold to move beyond current notions of mobile and collaborative learning: Can students in these diverse, distanced contexts learn by learning to work together? What might be provoked by these interactions, which would not otherwise occur within each context?

This paper describes the design and evaluation of the 'Out There and In Here' (OTIH) system and activity. Through this we contribute an example of the emerging potential of ubi-learning systems. OTIH is designed to support live collaboration between higher education students based in a technologically-enhanced room, and their peers engaged in scientific fieldwork at a distanced site. Using data from a pilot and two full day field trials, this paper explores the interactions between the two diverse groups, and the experience of those at each location. We shed light on the potential value in such systems and identify major issues in supporting these kinds of interactions.

\section{BACKGROUND}

\subsection{Dialogues between Diverse Situations}

A central concept of this work is that value can be gained by connecting users across diverse 
situations. As Olson \& Olson (2000) argue, the affective potential of users' locations is clear, and even with new technologies, distance still matters. A corresponding view is that connecting those with situational differences often leads to the clashing or merging of the disparate perspectives, which are the result of their unique experiences, capabilities and access to resources. Fischer (2005) argues that the spatial, technological and temporal distances between connected people, along with diversity, are sources of social creativity. In education, Wegerif (2007) argues that activities based on encouraging dialogue, questioning and redefining students' understanding, are appropriate to teaching key skills such as reflection, creativity and learning to learn. This 'Dialogic' approach is in convergence with many of the possibilities of new technologies, which are valueable because they change the perceived nature of space and time and can hold "two or more perspectives together in tension”, opening up the 'dialogic space' (pg. 4).

There are many possible approaches to connecting users to another place: Noting the combined potential of remote communications and robotics, Minsky (1980) envisioned 'Telepresence' technologies allowing remote operators to observe and act in another location. He defined a major challenge as achieving the sense of "being there". Whilst telepresence and other CMC technologies often aim to replicate rich, natural interactions, Hollan \& Stornetta (1992) argued that this aim has often limited thinking: New tools can be most valuable when they support activities that shared presence does not. Our focus here is not to attempt to transpose fieldwork or indoor learning through technology. Instead it is to bring those involved in disparate activities into a shared dialogic space.

In framing this design space, it is important to consider analogous systems where similar interactions are supported. In situations that link field-based and indoor collaborators such as space exploration or the management of transport networks it is common to find a 'control room' type environment. These rooms have common features across domains: Awareness information is displayed on walls; workstations allow individual work whilst co-located; multiple channels of communication are available, along with access to information resources; there is often a central table to support group discussions using maps and documents. Studying the London Underground control room, Luff \& Heath (1998) noted that the mobility of collaborators has received little attention in CSCW. They identified that problems arise from the inability of staff to effectively access relevant information when away from the control room.

The concept of a 'Collaboratory' is also relevant. Bos et al (2007) describe a wide range of systems under this umbrella term, from shared data sets to systems that provide shared access to research tools such as a telescope. They note that at least a secondary purpose of many collaboratories is training. While collaboratories generally operate over the long term, we aim to support immediate, live interactions. Where collaboratories aim to harness cross-organisational potential, our aim is to leverage the capabilities of collaborators in the field with others better able to perform information seeking, analysis and reflection tasks.

\subsection{Field, Mobile and Collaborative Learning}

Fieldwork is an important part of education and practice in many domains, particularly in the natural and social sciences. It provides uniquely valuable, practical experiences beyond the classroom. For example Elkins \& Elkins (2007) identified that a significant increase in conceptual understanding occurs through field-based courses in earth science. They attribute this to the ability of fieldwork to link theory and practice. Due to its roaming nature, fieldwork has stood out as an area where mobile technologies could be useful, and mobile learning is becoming integrated in to the mainstream. Through the iterative design and evaluation of LillyPad, Rogers et al (2009) explored the potential to support sensemaking in the field by providing mobile support for information seeking, data collection and analysis. The Personal Inquiry project developed software to guide students through the generic process of a scientific inquiry, whilst allowing them to focus on topics of personal interest. Evidence suggests that this approach had motivational benefits (Collins et al, 2009).

Building on this, some prior research has identified the potential to connect indoor and mobile activities: Rogers et al (2005) describe a vision of 'ubi-learning' that includes sharing and commenting on of field data at a distance, and the use of interactive tabletops in public settings to show data collected by community members. In research as well as education, data collected in the field is generally analysed and reflected upon through dialogue after the event, directing further fieldwork in iterative cycles. Yeh et al (2006) found that biology researchers viewed time in the field as a limited commodity, and therefore systems that distract from interaction with the environment are unlikely to be utilised. Potential benefits to the approach explored here could therefore include: Allowing students in the field to focus on the environment, whilst students indoors perform information seeking and analysis tasks, teaching communication and collaboration skills, and making fieldwork inquiries more efficient.

Joel et al (2004) explored this potential to link schoolchildren in class and field, identifying a need 
to design suitable activities alongside technologies. As a solution to the complexity of the potential collaboration between children, a structured approach with defined roles was found to be appropriate. These include 'Data Gatherer' and 'Scout' in the field, and 'Director' and 'Task Manager' in class. In further exploration of this approach, Kurti et al (2008) designed game-like activities and found evidence that the context of the learning did impact on how children interpreted and dealt with information.

While these designs focus on structured interactions, education research suggests that loose 'Collaborative Learning' approaches could be more appropriate for older students, as they utilise pre-existing social skills and motivation. McWhaw et al (2003) define this as situations where there is interdependence designed in to the activity, but where the students are given control to direct and structure their approach. This is contrasted with Cooperative Learning, where the interdependence is within a well-defined structure controlled by educators. Both forms are found to be beneficial to improving communication and social skills, particularly if they bring together diverse people.

\section{FORMATIVE RESEARCH}

To further understand this area, we elicited data on the nature of fieldwork, and its place in higher education earth science. The methods used were posters placed in a faculty common room, asking for written responses on the value of fieldwork, followed by a workshop with 19 educators and postgraduates. We were also influenced by an existing project: Enabling Remote Activity (ERA).

Fieldwork in the earth sciences - and more specifically in geology where our main trials took place - involves visiting sites with visible geological phenomena such as quarries or cliff faces to examine aspects of the earth. These include rock types, fossils and sedimentary layers. Guided by tutors, students undertake a range of tasks within an investigation including observing, identification, sketching, measuring, mapping, recording data, using secondary sources, data analysis, interpretation, drawing conclusions and reporting. Figure 1 shows some of the contrasting forms of information a student will interact with. An essential learning outcome at this level is the integration of several lines of evidence to formulate hypotheses. This involves making connections between information from different contexts, and dealing with real world complexity in considering what could be inferred. Educators noted that students struggled to learn to develop hypotheses that effectively used the evidence at hand, either in the field or otherwise. The integration of fieldwork, analysis and theoretical understanding are considered key to developing this skill.

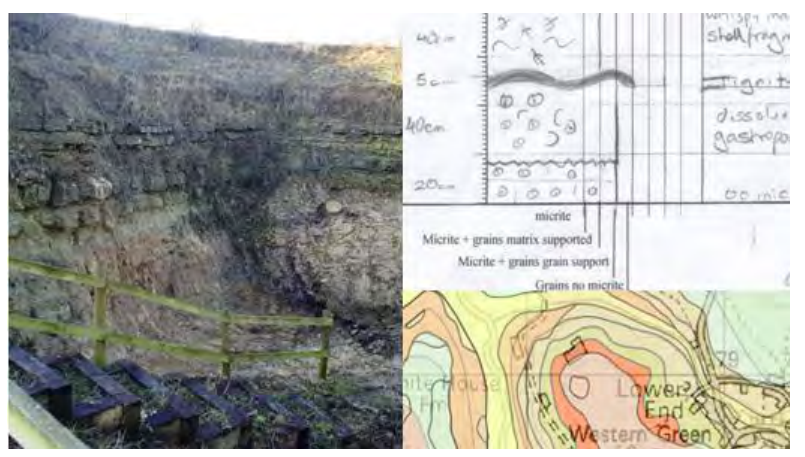

Figure 1: Physical interaction in the field contrasts with analytical map reading and log creation.

The ERA project was an inspiration to this research. This developed technology to connect geology field sites with distanced learners. These have now been integrated into actual courses. The implications from trials included that students unable to take part in fieldwork could be empowered through having remote access, and could complete aspects of geology fieldwork through an in-situ guide. Concurrent remote analysis of data was perceived as providing a beneficial experience that informed field-based students. Although remote experiences could not fully replicate field-based experiences, ERA highlighted potential to improve experiences at both locations (Davies \& Bartlett, 2007).

\section{DESIGNING 'OUT THERE AND IN HERE'}

The formative research guided the development of scenarios in which potential designs could be understood. We then expanded upon these ideas through a design workshop, bringing together an experienced geology tutor with a user experience consultant and the research team to develop storyboards and define an initial design.

\subsection{Conceptual Design}

We began the project with some essential concepts: That there would be two groups of students: One based 'Out There' in the field and engaged in activities that were largely similar to a standard field trip, such as observing, collecting measurements, making notes and developing ideas about the site. Another based 'In Here', able to interact with those 'Out There' with a view to gaining a valuable learning experience. The interaction should also be balanced, so that the 'In Here' team would reciprocate by performing analysis and sensemaking, taking advantage of the resources and technology at their disposal to find and share relevant information. 
The formative research suggested that social interactions were another important facet to the fieldwork experience. Whilst interaction between locations was essential to the premise of this exploration, it was also important that the students could interact effectively within their location. As discussed, there was potential for technology to detract from the field experience for those 'Out There', as well as overload the 'In Here' team with information. Rather than continuous telepresence, interaction was to occur at the discretion of the users, with guidance in the form of a timetable. Considering this, the notion of being 'live' was the focus - reflecting an activity that is happening in both locations simultaneously, but without constant interactions - rather than notions of 'being there'.

\subsection{Activity Design}

The design of a suitable learning activity was a strong theme throughout our discussions. To develop collaboration and communication skills, the approach in this design was to allow roles and patterns of interaction to be defined by the students. A base level of interdependency and shared structure was deemed to be required so that the teams at each end would feel compelled to interact, but we also wanted this to encourage creativity from the students, and freedom for the tutors to direct the activity as they saw fit.

An experienced tutor was to be based with each group. The two tutors decided upon suitable goals involving interdependencies between the groups, for example the 'In Here' team were to create a log representation of the geological bed layers based on incoming data. The groups would share photos and information to develop an understanding of the site. In addition, the formative research highlighted that the development and evaluation of hypotheses would be an appropriate shared structure to encourage dialogue between and within the teams: Through a process of making explicit their ideas, relating them to evidence, and deciding how confident they were in them, it was hoped that the students in both locations would share their understanding and improve their inquiry skills.

\subsection{System Design}

The OTIH system consists of a combination of devices and software utilised across the two locations. A key concept is that - though users at each location have access to different resources and use different devices - there should be a base set of shared functionality available to them to interact together effectively, whilst utilising their specific circumstances. To this end, a web-based content management system (CMS) was developed as a basis for information sharing between the two sites. This can be interacted with through any device with a web browser. For awareness purposes, the CMS homepage displays the 'Latest' view of images, hypotheses and votes recently added to the system. Users can then review and annotate images, develop and vote on hypotheses, and view a timetable for the day. The system provides an interface using standard HTML forms that would be familiar to anyone with experience of using the web.

\subsection{1: 'In Here' Technologies}

Users 'In Here' can interact with the CMS, as well as access online resources and use instant messaging software (IM) through two laptops. To add documents to the CMS, users can drag or copy files into personal folders on the laptops.

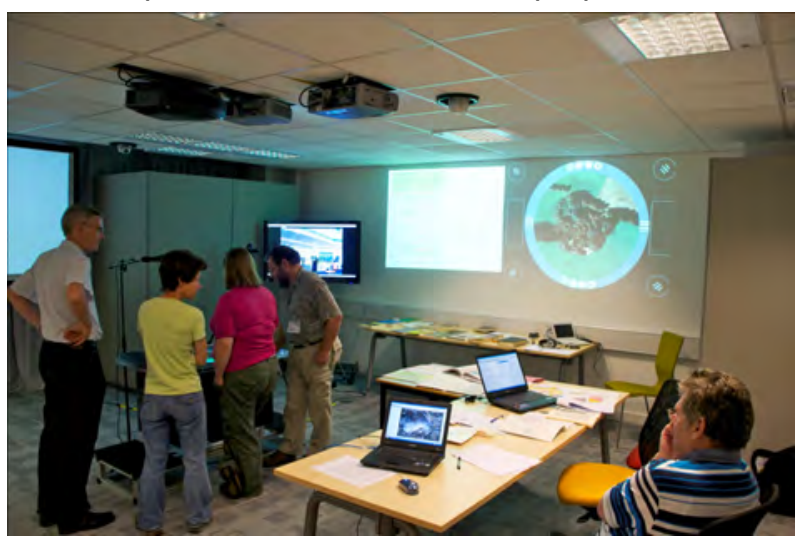

Figure 2: The 'In Here' Environment

The centrepiece of the environment is a Microsoft Surface tabletop computer, running a bespoke application. The system has four modes: i) Map, which provides access to satellite maps from an online server, and shows the current location of 'Out There' devices through their GPS information. The map mode is also used to view photos added from each mobile device ii) Viewer, which supports shared viewing of documents such as photos and geological maps (see figure 3), iii) Hypothesis Evidence, supporting the development and discussion of hypotheses and adding documents as evidence, and iv) Hypothesis Vote, which allows the users to vote on their confidence in a hypothesis and see the votes of others. Individual tagged object tokens are used to identify each student, in order that they could collect files of interest, and vote individually on hypotheses.

A mirror of the tabletop display is projected on to the wall of the room, along with other projections showing the 'Latest View' from the OTIH CMS, and the timetable for the day (see figure 2). A desk area was provided with laptops for accessing the web, specialised geology resources and communication via instant messaging, as well as the OTIH CMS. A further table housed a wide range of non-digital earth science resources such as books and maps. 
A speakerphone and scanner / digital camera was also provided in the room.

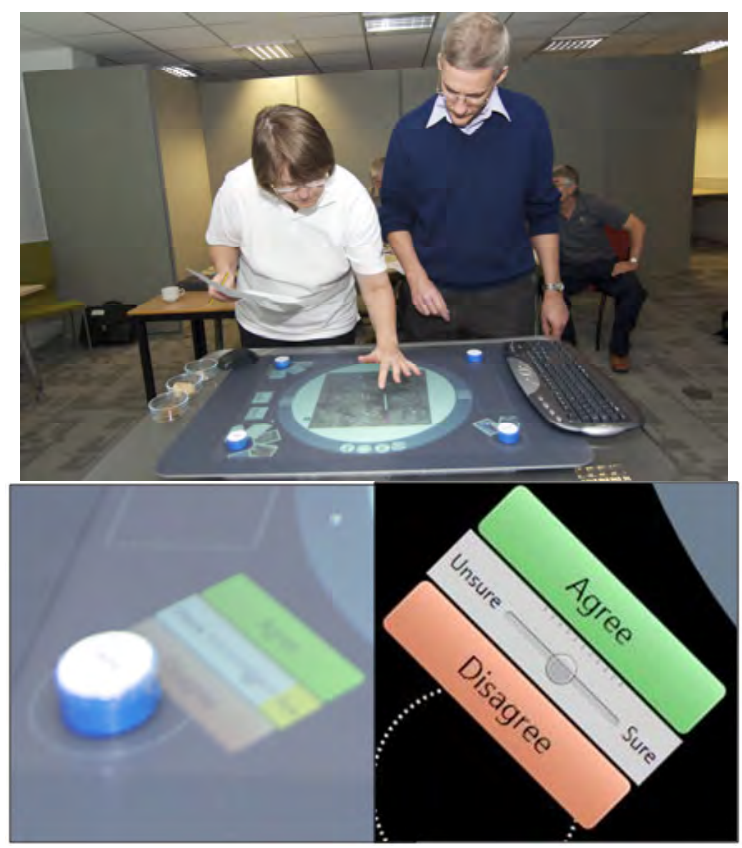

Figure 3: Tabletop computer being used for discussion of incoming images (above) and voting interface using personal tokens (below).

\subsection{2. 'Out There' Technologies}

At the field site, Android-based smartphones and an Apple iPad tablet computer are connected to this network, allowing students to take photos, communicate using IM, and access the OTIH CMS to view documents, develop and view hypotheses, and vote. Software is integrated with the camera application on the Smartphone devices so that users can easily upload photos to the CMS. A static video camera was located to show a wide view of the field site, which was streamed back to 'In Here'.

\begin{tabular}{|c|c|c|}
\hline \multirow{2}{*}{ Vlaw Ecill } & \multirow{2}{*}{\multicolumn{2}{|c|}{$\begin{array}{l}\text { User a agrees, and is very sure } \\
\text { User b agrees, and is neither sure nor } \\
\text { unsure }\end{array}$}} \\
\hline & & \\
\hline Predominantly carbonate deposition near & Userd has no & $\begin{array}{l}\text { I voted } \\
\text { voted }\end{array}$ \\
\hline $\begin{array}{l}\text { Shore with oscillating periods of formation of } \\
\text { ooliths and shell beds. Intermittent minor }\end{array}$ & Add or Change You & otes: \\
\hline $\begin{array}{l}\text { disconformities probably marked by relative } \\
\text { sea level changes. Oscillating energy }\end{array}$ & User $x$ & Usery \\
\hline conditions. Marls could be deposiled in & No Vote $[\mathrm{D}$ & No Vote 1 \\
\hline lagoonal environment & Your Vote & Your Vote \\
\hline Evidence: & $4 \quad 10$ & $4 \quad 1$ \\
\hline 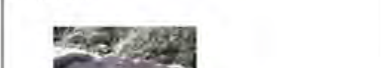 & $\begin{array}{l}\text { Contidence: From 0 } \\
\text { e Unsure to } 10= \\
\text { Sure }\end{array}$ & $\begin{array}{l}\text { Confidence: From } 0 \\
=\text { Unsure to } 10= \\
\text { Sure }\end{array}$ \\
\hline & Submit Vote & submit Vote \\
\hline
\end{tabular}

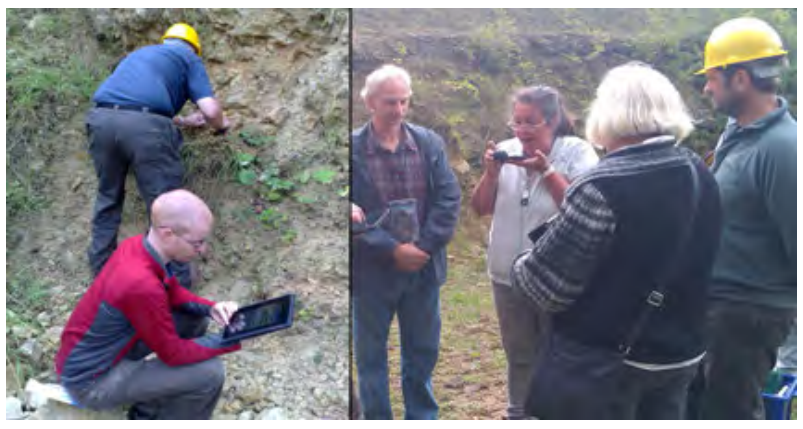

Figure 4: Mobile interface (above) and use of tablet computer and Smartphone at the field site (below).

Suitable networking solutions are dictated by local conditions at a field site. In the trials discussed here, a local Wi-Fi network and server was set up at the quarry, which then used $3 G$ mobile broadband to connect back to the 'In Here' site. This allowed the best possible connectivity for the mobile devices as the $3 G$ modem could be positioned on high ground above the quarry. In other locations with more consistent $3 G$ connectivity, we have connected smartphones and mobile video cameras directly through individual $3 \mathrm{G}$ connections. This can reduce the amount of hardware and setup time, but the local Wi-Fi solution has advantages for both consistent connectivity and supports local caching of image files to improve the responsiveness of the system.

\section{EVALUATIONS}

\subsection{Trials}

Naturalistic, in situ evaluations of the system were identified as the effective way to understand the real world potential for this kind of collaborative learning activity, and to identify issues with the design. OTIH has been the subject of three such trials to date. Firstly we carried out a pilot trial with 4 participants (2 in each location) performing an evidence gathering and hypothesis development activity based in a local nature trail. This allowed us to identify issues with our initial system, leading to the current design described in section 4.3. Two full day trials of the system with a total of 21 participants were then performed. Both trials followed the same form, and used current higher education earth science students and experienced tutors. In each case a group of 4 students along with a tutor were stationed in the 'In Here' environment, while another set of students (6 in the first trial and 5 in the second) were taken to a field site - a disused quarry of interest approximately 10 miles away - accompanied by another tutor.

A basic timetable for the trials was designed by the tutors: All the students met initially at the 'In Here' room, as it was hoped this would build familiarity between the students and allow everyone to 
understand the 'In Here' environment. After an explanation of the system and activity, the 'Out There' team travelled to the field site, while the In Here team used this time to gather information about the field site and wider area. On arrival, the teams would initiate communications which would continue as they felt appropriate, with the fieldbased team asked to perform an investigation of the site, sending information to the 'In Here' team, who used this for analysis and to inform further information seeking. At the end of the day, the field team returned to the In Here room for discussions.

The complexities of running this kind of activity are non-trivial, and will be discussed further in the findings. The first of the full trials suffered from a networking problem that resulted in some delays to image sharing, and a failure to receive the video stream 'In Here'. In the second trial, the system worked as intended. Both trials produced rich qualitative data about the subjective experiences of students and tutors, as well as examples of the kind of interactions and outcomes that are possible through connecting across these diverse situations.

\subsection{Analysis}

Researchers were present in both locations to observe interactions. Audio and video of the In Here environment was recorded throughout the day. At the field site, a mobile researcher filmed throughout the two trials. Initial background questionnaires were collected, and at the end of the day, the two groups of students took part in separate focus groups to discuss their experiences. The tutors also attended feedback sessions after each trial, as well as training sessions and meetings prior to the trials to discuss their role and develop their understanding of the technology. The focus of these exploratory evaluations was rich data collection in situ. We focus on qualitative data of the participants' experiences, and a log analysis of interactions within and between the groups.

\section{FINDINGS}

Table 1 quantifies the main communications between the groups in the full day trials. In both trials, it was found that the 'Out There' groups uploaded the majority of images $(76 \%$ across the two trials). These were a conduit and record of much of the information gathered in the field. In Here students added some images scanned from books and maps, and also screenshots from the specialist online mapping system Digimap. The development of hypotheses and voting played a more significant role for the 'In Here' groups, although this is only visibly the case in this data in the second trial, where they generated more and longer hypotheses, and each voted at least once on all the hypotheses produced.
Table 1: Interactions between the teams in the two fullday trials

\begin{tabular}{|l|l|l|l|l|}
\hline Trial 1: & $\begin{array}{l}\text { Images } \\
\text { Added }\end{array}$ & $\begin{array}{l}\text { Hypotheses } \\
\text { Developed } \\
\text { (avg. length } \\
\text { in words) }\end{array}$ & $\begin{array}{l}\text { Votes } \\
\text { Cast }\end{array}$ & $\begin{array}{l}\text { Voice } \\
\text { Calls (avg. } \\
\text { length in } \\
\text { minutes) }\end{array}$ \\
\hline In Here & 7 & $2(11)$ & 9 & $4(9.3)$ \\
\hline Out There & 23 & $2(13.5)$ & 7 & \\
\hline Trial 2: & --- & --- & --- & --- \\
\hline In Here & 11 & $5(23.4)$ & 32 & $6(5.3)$ \\
\hline Out There & 33 & $2(13)$ & 7 & \\
\hline
\end{tabular}

Distinctions between the trials can be explained by both connection issues between the sites on the first trial, additional technology provided in the second (the tablet computer in the field, and a scanner rather than a digital camera for more efficient use of non-digital resources at the 'In Here' environment). There were also improvements in the tutors' understanding of how to run the activity and utilise the system. More images, hypotheses and votes were shared in the second trial. A greater number of phone calls also occurred in the second trial, but the total length of time spent in phone conversations was higher in the first, potentially due to difficulties with other channels (e.g. users found IM easier on the tablet than on phones).

In the following sections we discuss our qualitative analysis and highlight several concepts of interest. Firstly we analyse how dialogue and the structuring of collaboration between the teams facilitated learning. We then consider issues around the experience of being connected. Finally we discuss the distinct benefits for learning at each location.

\subsection{Dialogue}

Our analysis found that most interactions consisted of the Out There team responding to direct questions from In Here, through voice calls and IM. An Out There participant stated that they "responded mainly to questions that were raised and it was only because there was a contention... (that they used documents sent from In Here)" Another Out There participant stated that "You couldn't keep up to date" with content added to the system due to the pressure to collect data. This shows that - given the elements competing for the attention of those Out There - an emphasis on supporting and encouraging dialogue should be an effective design approach for enabling effective learning in this kind of activity. In this approach, it is the task of those In Here to focus the activities of those Out There, rather than simply adding relevant information to the CMS.

As an example of designing for dialogue, hypothesis development was generally effective in encouraging discussions within and between the 
teams. More attention and time was spent on developing hypotheses In Here, which can be explained in part by the focus on analytical thinking. Time pressures and engagement in the field site meant that the Out There team sometimes had to be prompted by tutors and In Here students to take part, but it can be argued that this was beneficial towards encouraging them to reflect. Whilst there was on one occasion a split in voting between the locations, there was little disharmony found in the voting within locations, as the teams spent significant time negotiating their hypotheses together until there was little or no dissent left.

There were clear cases where document sharing led to the development of novel understanding. This was mainly prompted by disagreements in the dialogue between the teams. Most dramatically in the second trial, a map added from 'In Here' and real world observations didn't match. The students had identified an error in the published data:

\section{New instant message received on the tablet}

F: "Just a digimap that shows that there is a faultline where you are standing with a downflow to the west" Pause while student reflects...

F: "That doesn't seem right... No that is the East they say downflow is to the East. They were trying to trick us." (laughter)

F: "Apparently they've sent us a digimap to show it." Participants pass the tablet around to view map. Tutor: "No that is definitely a downflow to the east. That must be a geologists' mistake..." Everyone Laughs. "We are about to rewrite the BGS [British Geological Survey] map. Everyone looks, discussion results in the students all giving a cheer and 'high 5' to each other.

The clearest value of the system was therefore its ability to provoke reflection and insight, through dialogue that made explicit the differences in understanding between the two groups. Points for reflection were identified through questions or viewing hypotheses from the other team, and occurred for varied reasons. In some cases it was simply because - as in the previous excerpt sources of information at each location were in disagreement. Another common cause was that information filtering did not explain the reasoning behind a statement. The following excerpt is from the In Here perspective, where a message provokes disquiet and further exploration because of a lack of context:

B: (reading an IM message from 'Out There' on a laptop about beds 9 and 10) "How do they know they are algae-eating gastropods?"

B ... "where do they get that?"

D (laughs): "I dont know, thats a leap."

Tutor: "wow" (sounding unconvinced)
Tutor: (zooms into image of the beds on the tabletop) "This is, that's bed 10, now" Students look at mirrored projection and discussion continues on the nature of beds 9 and 10.

The 'In Here' group then focused their attention on analysing the rock bed concerned. This contention was remembered and was the topic of further dialogue at the end of the day meeting.

\subsection{Structuring Collaboration}

The loosely-structured collaborative learning approach provoked creative thought on how to work together, but also frustration in overcoming problems introduced by distance. Methods through which to collaborate were discussed extensively. Attempts to empathise towards those in a situation with a very different set of foci and resources resulted in the common observation of 'information filtering' processes - reflecting on and distilling from the large mass of potential data available, that which was expected to be of interest at the other location. As the In Here tutor described in post-trial discussions, "information was being filtered for us". For example on finding a point of interest a student in trial 2 noted that: "This will be interesting because I don't know which one (photo) we want to send back, or how many we want to send back". In Here students also perform similar filtering using the resources at their disposal, for example finding images of local maps, communicating information about the area, and providing information with which to identify fossils they expected to be found in the area.

Whilst it could be effective in teaching collaboration and communication skills, it is clear that the nature of the activity means that certain tasks will only be performed by a student at one location or the other. E.g. only the students in the field actually took measurements of the rocks and labelled the beds. The In Here tutor noted in post-trial discussions that "finding it out in the field requires a lot of working out with original observations". In this sense the system does not replicate field learning for those indoors, or indoor learning for those outdoors. However the ability to distil, communicate and work together effectively using these forms of information is a valuable learning outcome that is not being achieved through other activities.

Analysis of the focus groups identified that varied understandings developed as to how the groups were meant to work together. An 'Out There' participant stated that: "(the 'In Here' team) want the information and that effectively means they have to be in the driving seat a little at least.", going on to say he expected more demands from In Here to carry out tasks than actually occurred. Another Out There participant from trial 2 stated that: "We 
did get input from (in) here, stuff that we couldn't necessarily find for ourselves when we were out there...but we were quite busy doing what we were supposed to be doing". Based on this, 'Out There' students expressed a concern that those 'In Here' might have felt undervalued, as there was not enough time to respond to all of their input. This was corroborated by the In Here focus groups, one participant stating that: "I don't know how the people out there, whether they actually used stuff that we sent" In future work, feedback mechanisms might provide awareness of what is being used, but this is not just a system design issue - the activity needs to support better understanding and utilisation in information sharing.

Some In Here participants expressed frustration and stated a desire for more structured roles to support communication between the teams. One stated that "one or two people whose jobs are to deal with communications to the In Team...to say, "Right, what have you got that you want me to ask or tell the other team?" or... "They've just sent us some pictures and a link off the internet."...would have made it so much better" students performed this roles dynamically, but it was clearly appealing to students that this process be structured. However, the current approach meant that all students had equal ability to take part in dialogue.

\subsection{Experiencing Live Connections}

It is interesting to explore how specific technology and interactions made the teams feel 'connected' with each other, and the potential value of this. The video stream was not considered to constitute effective telepresence as the resolution was too low to transmit detailed information. However, seeing their peers in the field engaged In Here students and provided up to date background such as the weather and the general nature of current activity. One participant stated that: "there was nothing really educational (in the video) but it did give us a slightly more emotional tie to them". The In Here tutor felt that a failing in the first trial was that no pictures were sent back that actually featured the team members on location. Whilst the detailed images of beds and samples that were shared were useful, on their own they do not differ significantly from an activity based around a static information resource. In both these findings, it is clear that conveying the sense of live interaction with actual peers is important.

Throughout the trials, participants' sense of time appeared to differ between the locations. A sense of momentum was achieved through timely, effective dialogue, and could be lost through a lack of rhythm in these interactions. In the previous excerpts, incoming communications focused the work of the In Here team. However, even in the more successful second trial, logistics meant that timing was often less than perfect, as the excerpt below shows:

Everyone at the Surface studying an image. Phone rings, $B$ answers, greets Out There (OT).

OT: "... you should have a close up of the rock face here and our hypothesis."

$A$ and $C$ look at the Latest Updates projection

$\mathrm{B}$ : "Uh we have a hypothesis that this is sedimentary rock, which we've agreed with, we've actually put up another hypothesis, which... we think its oolitic limestone"

OT laughs: "Great!"

B: "Can you see our hypothesis, that we sent you?" OT: "Uh yes we were just voting now...just doing that as we started to call"...

Pleasantries are exchanged and call ended.

B: "God they are so slow out there!" laughs

Students return to looking at images on the Surface

This example shows the complexity of orchestrating an activity between the sites so as to effectively maintain momentum. Fieldwork by its nature consumes time through travel, setting up equipment and collection of data. The 'Out There' tutor decides to make a call to make the In Here team aware that they are moving. However, the 'In Here' team have been waiting for responses to their hypotheses, and hoped that they were ready to send more photos and information. This and other data sources show an asymmetry, as the quality of the 'In Here' students' experience is more dependent on interaction with the 'Out There' team than vice versa. Consequentially the 'Out There' team could - through empathising with this situation - become pressured to deliver. The tutor based in the field fed back that at some points she was "stressed" by feeling that "If we don't get the material back they are stuck". Analysis of videos also identified several incidents where field-based participants were conscious of the reliance of the In Here team on them:

$\mathrm{E}$ : (worried that they have not responded to a hypothesis from 'In Here') "Do we need to text them and say we agree with your hypothesis?" ...

$\mathrm{H}$ : "Can we give them a call so we can tell them?"

Tutor: "We are going to call them in just a minute" $\mathrm{H}$ : 'I can imagine they are probably quite frustrated sitting in there.'

It is clear that - if harnessed correctly - this connection can be very motivational for students in the field. However this needs to be balanced with the aim of engaging students with the environment.

\subsection{Benefits for Learning}

In the post-evaluation focus groups, all participants agreed that they had in some way taken part in a 
useful learning experience. Opinions contrasted as to how and why this was the case:

For those in the field, there was agreement that this was significantly different to a standard field trip. As mentioned previously, students were unsure about the nature of the interactions aimed for. They could see the motivation in "being the eyes and ears of those in the field", and noted that "their (In Here) on-going hypothesis development is important to what you're thinking" One highlighted the value of being able to reflect both during and after the field trip, stating that "I can see with all this stuff being put up back here, the pace of any field day doesn't allow you really to appreciate (what was found)".

In considering the 'In Here' experience, a participant in the first trial suggested it was "good to see how other people work and approach a problem...it does help and support problem-solving skills because we were able to work as a team...to find the information that we were trying to pass onto the other team". This highlights the value of combining the context of a live field investigation with work with co-located students. In the second trial, a participant reflected that: "I don't think it replaces a field trip in experience but I think its... finding other ways of working where it's valuable.".

\section{IMPLICATIONS FOR DESIGN}

Whilst the Out There experience could be considered as an augmented field trip, the experience In Here was a highly novel learning activity with potential to bring the complexity of real field inquiries indoors. In both cases, there is evidence to suggest that communication and collaboration skills are being developed, as well as methods of performing inquiry and using new technologies. In designing for these experiences, conceptual goals such as encouraging dialogue and collaboration are best achieved through taking a holistic approach that considers the complex relationships between the system and activity.

There were some cases where the trials highlighted that support for representation was inadequate. In particular, the 'In Here' team felt that they should be able to communicate their analyses through a multimedia collage, with a participant stating that: "we would have liked to have sort of juxtaposed pictures together", going on to state that "I think we needed to build our own collage and we couldn't." An 'Out There' participant noted that, in relation to voting: "you couldn't capture (responses to hypotheses) in such a simple 'yes' or 'no'”. A related issue is supporting In Here students to effectively build an understanding of the field site. Despite efforts by field-based students to label rock faces and beds, and produce wide view photos of these, students 'In Here' found it difficult to visualise where data was located. An 'In Here' participant stated that "you've got a lot of photos with little details...and then you've got...the wide overview and you need to kind of place (the photos)...you didn't have that $3 D$ aspect in the photograph, you also didn't have scale."

Future designs should consider how activities such as the development of hypotheses and analyses could be better conceptualised as a rich dialogue both between and within the teams. In these trials functionality to explicitly post revisions, counterarguments, and complex relationships could have provoked further dialogue. This could become a narrative of the development of understanding between the teams. Such an approach could build upon systems for 'argumentation' such as Cohere (Buckingham Shum, 2008), by placing these in the context of live fieldwork inquiry.

Rather than attempting to replicate the experience of the other team, we used connectivity to support dialogue and collaboration that would be beneficial to both teams in different ways. The analysis of the trials highlights successes, but also difficulties due to the imbalances between the abilities and reliance of the teams on each other. In particular, 'In Here' students are much more reliant on timely interactions from those Out There than vice versa, whilst - in order to fulfil this need - those in the field have to balance engagement with both the environment and the technology. Imbalances are also to be expected in the use of the shared system - 'Out There' groups produced more photographic data whilst 'In Here' groups spent more time on hypotheses. These differences need not be problematic, but the system and activities need to be structured such that the input from both ends is valued rather than overlooked, as this may result in a lack of belief in the value of students' own efforts.

The amount and type of structure designed to encourage interactions between the groups is a complex topic, but one to which some understanding has been reached here: Encouraging dialogue between the groups is a positive approach because it explicitly prompts for responses from the other team, and highlights differences in understanding. Evidence was found in the trials that collaborative learning approaches have value. This put the onus on students to organise themselves whilst empathising, discussing and reflecting with their co-located team. Although students found this difficult at times, it could be a valuable learning process, and many issues of this kind are faced in the modern workplace. More task management and situational awareness functionality could improve the ability of distanced teams such as these to self-organise. 
In designing this type of experience, both interlocation and intra-location social interactions are important. Aside from the value of connecting the teams to each other, the trials highlighted the value found in having co-located teams at each site, rather than distanced individuals. There was evidence that the experience of face-to-face discussions and seeing others perform tasks was highly valued by the students. At the same time, the groups in each location were both motivated and directed by the existence and actions of the other team. Whilst we have yet to fully define the specific value of a live activity and connection, it is clear that this can produce a highly novel learning experience by juxtaposing an authentic field inquiry with an indoor context more commonly used for analytical and conceptual learning. Evidence from the trials suggests that this led to results that would not otherwise be achieved.

While previous research identified the potential for children to interact through analogous systems, we have shown that this approach has particular value for $\mathrm{HE}$ fieldwork, and perhaps also more widely for cutting edge field-based research. Whilst mobile learning technologies have much to offer, this approach extends this value and could be advantageous where those in the field could direct too much attention towards technology, rather than their environment, for tasks that could be performed elsewhere. It could be advantageous in situations - both in learning or research - where insitu data collection and analysis could be performed concurrently, but are not because of the distances between field and laboratory. Finally, it is also suited to widening participation in all kinds of fieldwork activities, so that those who cannot be at the field can still gain some benefit.

\section{CONCLUSIONS}

This paper has highlighted how ubiquitous technologies present opportunities for extended forms of dialogue across diverse contexts, and that these are potentially valuable as learning activities. We have contributed an example system, and through evaluations, an understanding of how to harness this potential. As these technologies become even more affordable and pervasive, it will become easier to set up distributed systems such as OTIH to support radically new collaborative learning scenarios. Further research is needed to clarify how best to design for phenomena such as asymmetrical dependencies between students, dialogic representations, the motivational value of being live, and the self-structuring of collaborations.

\section{REFERENCES}

Bos, N., Zimmerman, A., Olson, J., Yew, J., Yerkie, J., Dahl, E. \& Olson, M., (2007) From Shared Databases to Communities of Practice: A Taxonomy of Collaboratories, Computer-Mediated Communications 12, 318-338. Blackwell. NJ.

Buckingham Shum, S., (2008). Cohere: Towards Web 2.0 Argumentation. 2nd Int. Conference on Computational Models of Argument, Toulouse.

Collins, T., Gaved, M., Mulholland, .P, Kerawalla, L., Twiner, A., Scanlon, E., Jones, A., Littleton, K., Conole, G. and Blake, C. (2008). Supporting location-based inquiry learning across school, field and home contexts. Proc. of MLearn '08.

Davies, S. \& Bartlett, J. (2007) Report for the Enabling Remote Activity project, JISC TechDis.

Elkins, J. T. \& Elkins, N. M. (2007). Teaching Geology in the Field: Significant Geoscience Concept Gains in Entirely Field-based Introductory Geology Courses, J. of Geoscience Education, 55:2. 126-132. NAGT.

Fischer, G., (2005) Distances and Diversity: Sources for Social Creativity. Creativity \& Cognition '05. pp. 128-136. ACM Press. New York.

Hollan, J. \& Stornetta, S. (1992) Beyond being there. ACM Conference on Human Factors in Computing Systems. 119-125. ACM Press, New York.

Joel, S., Arnott, L., Hine., N., Ingvarsson, S., Rentoul, R. \& Schofield, S., (2004) A Framework for Analysing Interactivity in a Remote Access Field Exploration System. IEEE Int. Conference on Systems, Man and Cybernetics. 2669-2674. IEEE Press.

Kurti, A., Spikol, D. \& Milrad, M. (2008) Bridging outdoors and indoors educational activities in schools with the support of mobile and positioning technologies, Int. J. Mobile Learning and Organisation, Vol. 2, No. 2, 166-186. Inderscience. Luff, P. \& Heath, C., (1998) Mobility in Collaboration, Proc. of CSCW '98, 305-314. ACM Press. New York.

McWhaw, K., Schnackenberg, H., Sclater, J., \& Abrami, P. C., (2003) From Cooperation to Collaboration: Helping Students Become Collaborative Learners. In R. Gilles \& A. Ashman (eds.), Cooperative Learning. Routledge.

Minsky, M. (1980). Telepresence. Omni Magazine, (June 1980) 45-51.

Olson, G. M. \& Olson, J. S., (2000) Distance Matters. Human-Computer Interaction vol. 15, issue 2-3. 139-178. Taylor \& Francis.

Rogers, Y., Connelly, K., Hazlewood, W., \& Tedesco, L. (2009). Enhancing learning: A study of how mobile devices can facilitate sensemaking. Personal and Ubiquitous Computing 14:2. 111-124. Rogers, Y., Price., S., Randell, C., Stanton Fraser, D., Weal, M. \& Fitzpatrick, G., (2005) Ubi-Learning Integrates Indoor and Outdoor Experiences. CACM vol. 48, no. 1. 55-59 ACM Press, New York. 
Roschelle, J. \& Pea, R. D. (2002). A walk on the WILD side: How wireless handhelds may change computer-supported collaborative learning. Int. Conference on Computer Support for Collaborative Learning. 51-60. Lawrence Erlbaum.

Wegerif, R., (2007) Dialogic Education and Technology. Springer Press, Berlin.
Yeh, R. B., Liao, C., Klemmer, S. R., Guimbretière, F., Lee, B., Kakaradov, B., Stamberger, J. \& Paepcke., A. (2006) ButterflyNet: A Mobile Capture and Access System for Field Biology Research. ACM Conference on Human Factors in Computing Systems. 1427-1436. ACM Press. New York. 